\title{
Index of drugs and other compounds
}

Acetohexamide 24I

Adenyl cyclase 218, 219

Adrenal corticosteroid(s) 222

Adrenaline 209, 2 I 9

$\beta$-Adrenergic blockers 209

Adrenocorticotrophic hormone 218, 219

Alcohol 209, 213, 233, 236, 240 see also Ethanol p-Aminobenzoic acid 205

Aminophylline 219

Aminopyrine N-demethylase 192

Aminopyrine 192, 233

p-Aminosalicylic acid 215, 216, 221 see also Aspirin, Salicylate, Sodium salicylate

Amitriptyline 219

Amphetamine 219, 242

Amphotericin B 217

Anabolic steroids 220

Aniline 233

Aniline $p$-hydroxylase 192

Antihistamines 236, 237, 241

Antipyrine 193, 200, 201, 233

Apisate see Diethyl propion

Ascorbic acid $195,231-236$

L-asparaginase 221

Aspirin 209, 214, 221, 236, 237 see also $p$-Aminosalicylic acid, Salicylate, Sodium salicylate

Aspirin hydrolase 192

Atromid see Clofibrate

6-Azauridine 221

Barbital 233

Barbiturates 195, 209, 234

Benzedrine see Amphetamine

Benzo( $\alpha$ pyrene $193,198,201$

Benzo(a)pyrene hydrolase 193,200

Benzodiazepine(s) 24I

Benzothiadiazine(s) 209, 212

Benzoyl-oxy-fenfluramine 242 see also Fenfluramine

3,4-Benzpyrene 233

Betamethasone 236, 237

Biguanide(s) 209, 219, 242

Biphenyl r94

Biphenyl-2-hydroxylase 192

Biphenyl-4-hydroxylase 192-195

Buclizine 24I

Butylated hydroxyanisole (BHA) 199-201

Butylated hydroxytoluene 200

Caffeine 209, $211,219,234$

Calcium salts $195,215,216,221$

Candicidin 217

Cantharidin 236
Carbon tetrachloride 195, 218, 237

Catecholamine(s) 21 8, 219

Celevac see Methyl cellulose

Cellucon see Methyl cellulose

Cerous chloride 218

Chenodeoxycholic acid 221

Chloramphenicol r94, 204

Chlorcyclizine 233

Chlordiazepoxide 24I

Chloretone 232, 233

Chloroform 218,234

2,2-bis(p-Chlorophenyl) - I, I, I - trichloroethane see DD'

Chloroquine 204, 236, 237

Chlorothalidone 200

Chlorothiazide 209, 212

Chlorphentermine 242

Chlorpromazine 209, 219, 222, 24I

Chlorpropamide 209, 212, 241

Chlortetracycline $203,215,216,221,241$ see also Tetracycline

Cholecalciferol 222

Cholesterol 236, 237

Cholestyramine resin $215,216,219,221$

Clofibrate 219-22 1

Coffee, instant 218

Colchicine 215,216, 221

Colestipol 216

Corticosteroids 218

Corticosterone 193,194

Corticotrophin 240

Cortisol 193, 209, 236, 237

Cortisone $236,237,24 \circ$

Co-trimoxazole $23 \mathrm{I}$

Coumarin(s) 209

Creatinine 204

Cyclic AMP 2,18

Cyclopropane 234

Cyproheptadine 24I

Cysteine 236

Cytochrome $b_{5}$ I 93

Cytochrome $P$-450 I9I-194, 197-I99, 201

Decorpa see Guar Gum

Deoxycorticosterone $23^{6}$

2-Deoxy-D-glucose $24^{\circ}$

Dexamethasone 209

Dexamphetamine 242

Dexedrine see Dexamphetamine

$\operatorname{Dextran}(\mathrm{s}) 22 \mathrm{I}$

DDT 197, 200, 201, 222

Dinabol see Methandienone

Diazepam 24I

Diazoxide 209, 2 I I, 2 I 2 
Dicoumarol 212

Diethylaminoethyl (DEAE) Sephadex 216

Diethyl propion 242

Diethylstilbestrol 236

Digitonin 236

Digoxin 206

Dihydrofolate 205

Dihydrofolate reductase 205

Dimethyl biguanide 209, 242

Dimethyl nitrosamine 195,218

Diphenadione $22 \mathrm{I}$

Diphenylhydantoin (DPH) 225-228, 233, 234

Diphenylhydramine 233

Drostanolone 220

Durabolin see Nandrolone

Duromine see Phentermine

L-Epinephrine $\mathbf{2 4 2}$

Ethanol 21 8, 222 see also Alcohol

Ether 234

Ethinyl oestradiol 220

Ethione 218

Ethylmorphine N-demethylase 193

Etiocholanone $23^{6}$

Fenfluramine 2x9, 221, 234, 235, 242

Ferritin 205

Ferrous sulphate 203

Filon see Phenmetrazine

5-Fluoro-3-hydroxymethylpyridine hydrochloride 2 I 9

5-Fluoro-nicotinic acid $2 \times 9$

FMS IA 242

Formiminoglutamic acid 205

Frusemide 209

Glibenclamide 209, 212, 241

Glucagon 209, 213, $219,221,242$

Glucocorticoid(s) $24^{\circ}$

Glucuronic acid I9I

Glucuronyl transferase 192

Glutathione 192

Glycine 191

Gold thioglucose 24r

Gonadotrophin 235

Growth hormone 193, 209, 218, 219, 222

Guar gum 24I

Haloperidol 221

Heparin 217

Hexobarbital 192, 198

Hexobarbitone 233

$\gamma$-Hexachlorocyclohexane 222

Hippuric acid 192

Histidine 205

Hydrazine 234

Hydroperoxide(s) 199

Hydroxybiphenyl 194

Indomethacin 215, 216

Insulin 209, 210, 212, 213, 217-219, 235, 240

Isoindole(s) 242

Isoprenaline $209,2 \mathrm{rg}$
Kanamycin 215, 216

Largactil see Chlorpromazine

Leptazol 234

Librium see Chlordiazepoxide

Lignin 216

Lindane $\mathbf{2 2 2}$

Liquid paraffin 2 I 5, 2 16

Lucofen see Chlorphentermine

Magnesium I95

Mazindol 242

Mebonazine 209

Medazepam 24I

Meprobate $\mathbf{2 3 3}$

Mepyramine 233, 237

Metformin see Dimethyl biguanide

Methandienone 240

Methotrexate 215, 216,23I

Methylamphetamine 234

Methyl cellulose 24I

Methylcholanthrene 233

Methyl testosterone 220, 221

4-Methyl umbelliferone glucuronyl transferase 192

Methylxanthine(s) 2 I 8

Monoamine oxidase inhibitors 209

Morphine 209

Nandrolone 240

Neomycin 204, 21 5, 216, 221

Nicotinamide 219,220

Nicotine 209

Nicotinic acid 209, 210, 219-22 I

Nilevar see Norethandrolone

$p$-Nitrobenzoate reductase 192, I94

$p$-Nitrobenzoic acid $\mathrm{I} 92$

Nobrium see Medazepam

Noradrenaline 219

L-Norepinephrine 24I

Norethandrolone 240

Norethindrone acetate $217,220,221$

Norethisterone acetate 220

Nortriptyline 200

Oestrogen(s) $217,220,222,235,240$

Oral contraceptives 209, 2 I 7, 222, 235

Orotic acid 218

Orphenadrine 233

Oxandrolone 217, 220

Oxyphenylbutazone 233

Paraffin, liquid 215, 216

Pentobarbital I93

Pentobarbitone I95, 233

Periactin see Cyproheptadine

Phenacetin 209

Phenethyl biguanide 209, 22 1, 242

Phenformin see Phenethyl biguanide

Phenindione 215, 216, 221

Phenmetrazine 242

Phenobarbital 192, 193, 198

Phenobarbitone 197, 199, 201, 225-228, 233 
Phenothiazine(s) 24I

Phentermine 242

Phentolamine 242

Phenylalanine 205

Phenylbutazone 193, 209, 212, 233

Phenyramidole 212

Phenytoin 204, 205, 209

Phosphodiesterase 2II, 218

Phosphorus, yellow 218

Phytosteral(s) 217

Polychlorinated biphenyl(s) 200

Ponderax see Fenfluramine

Prednisolone 237

Prednisone 236,237

Preludin see Phenmetrazine

Premarin see Ethinyl oestradiol

Primidone 225, 227

Probenecid 209

Progesterone $193,236,237,240$

Progestogen 222

Propanolol 209, 219, 241

Prostaglandin $\mathrm{E}_{1} 219,242$

Protamine sulphate 217

Protamine zinc insulin 240

Psyllium seed colloid 216

Puromycin 218

B-Pyridyl carbinol 210, 2 I9

Pyrimethamine 205

Pyrimidine-giymidine 24I

Quinine 20I

Reserpine 24I

Ronicol see $\beta$-Pyridyl carbinol

S992 242

Salbutamol 242

Salicylate 212,2 ro see also $p$-Aminosalicylic acid, Aspirin, Sodium salicylate

$\beta$-Sitosterol $216,2 \times 7$

SKF 525 A 197

Sodium salicylate $24 \mathrm{I}$ see also p-Aminosalicylic acid, Aspirin, Salicylate

Stanozolol 220

Stilboestrol 240 see also Diethylstilbestrol

Streptolysin O 237
Strychnine 194, 234

Sucrose 195, 218

Sulfinpyrazone $\mathbf{2 3 3}$

Sulphaguanidine 217

Sulphapenazol 212

Sulphapyrazone 221

Sulphonamide(s) 209, 217, 241

Sulphonamide sulphamethoxazole 205

Sulphonylurea(s) 209, 212, 213, 219, 24I

Tangeretin 200

Tenuate see Diethyl propion

Teronac see Mazindol

Testosterone 236

Tetrachloroethylene 204

Tetracycline 203, 204, 218,231 see also Chlortetracycline

Tetrahydrofolate 205

Theophylline 2 I 9

'Thiouracil 222

Thyroid hormones 220, 241

Thyrotropin 236

Thyroxine 219, 221

a-Tocopherol 195

Tolazamide 212,241

Tolbutamide 204, 209, 212, 241

Transferrin 205

Trifluperidol 221

Triglyceride lipase 218

Trimethoprim 204, 205

Tryptophan 221

Tyrosine 205

Ultra-violet irradiation 236,237

4-Umbelliferone glucuronyl transferase I 94

Uric acid 204

Valium see Diazepam

Vitamin A 236, 237

Vitamin D 222

X-irradiation 236

Zinc 195

Zoxazolamine I94, 233 